Egyptian

Orthodontic Journal

\title{
EVALUATING THE SAGITTAL BASE RELATIONSHIP IN AN EGYPTIAN POPULATION SAMPLE USING THE BETA ANGLE
}

\author{
Hanan A. Ismail*
}

ABSTRACT

Cephalometric analysis using the $\mathcal{A N} B$ angle and the "Wits appraisal" to diagnose sagittal base relationship can be very deceiving due to the many factors affecting these measurements and camouflaging the real relationship. The Beta angle is independent on any cranial land marks and gives a better picture of the sagittal base relationship. The aim of this study was to find the mean and cut off points of this angle (previously developed at Tufts university) in an Egyptian Population sample. 120 patient's files were selected and screened on the base of 3 criteria: angle $\mathcal{A N B}$, "Wits appraisal" and XY axis to differentiate between classes I, II and III Skeletal patterns.

Using ANOVA and Posthox analysis the mean value of Beta angle for class I was 32.21 degrees for class II 25.1 degrees and for class III 41.8 degrees. The Receiving Operator Characteristic curve analysis showed that the cut off point between skeletal classes I and II was $29^{\circ}$ and between skeletal classes II and III was $34^{\circ}$.

\section{INTRODUCTION}

The many analysis designed by the various contributors to cephalometrics attempt to evaluate the effects of growth and treatment mechanics on the patient. The challenge to the science of cephalometrics rests in the fact that it is not and never can be an exact science. However, it does appear that certain techniques can be refined to allow us to derive all the information that our cephalograms contain and in many ways hide from us. ${ }^{(1)}$

\footnotetext{
* Assistant Professor of Orthodontics, Faculty of Dentistry, Alexandria University.
} 
Evaluating the sagittal apical base relationship is of great importance in orthodontic diagnosis and treatment planning. Although the ANB angle is very popular and useful in evaluating the jaw relation it has been pointed out by many authors that in some cases it does not accurately describe this relation. This is due to variation of the cranial and facial skeleton..$^{(1-5)}$

Jacobson $^{(3)}$ demonstrated the qualitative effects of the jaw rotation and the length of the anterior cranial base on the angle ANB. Binder ${ }^{(6)}$ Showed that the vertical displacement of point $\mathrm{N}$ in an upward or a downward direction will increase or decrease the ANB angle respectively if the positions of points A and B are fixed.

Hussels and Nanda ${ }^{(7)}$ analysed the factors affecting the ANB angle and added to the previous three factors the vertical growth reflected in the distance between $\mathrm{N}$ and $\mathrm{B}$. A geometric triangle shows that the longer the lines are extended from an angle, the further apart they become in relation to each other. Because of this, it is possible for two patients to have the identical ANB values but a different horizontal distance between points $\mathrm{A}$ and $\mathrm{B}$ since the length of their faces may vary. ${ }^{(1)}$

Thus the following factors ${ }^{(7)}$ were identified to affect the ANB angle (fig.1):

1-Growth rotation of the jaws, 2-vertical growth reflected in the distance between points $\mathrm{A}$ and $\mathrm{B}, 3$-vertical growth reflected in the distance between $\mathrm{N}$ and $\mathrm{B}$, and 4-length of the anterior cranial base and the anteroposterior position of the nasion.

Researchers have tried to find other methods for evaluating the apical base relation. Stoner and his colleagues used linear measurements in 1956 to study Tweeds cases by dropping a perpendicular from A and B to the Frankfort horizontal plane to measure the horizontal distance between them. In 1969 Taylor measured growth changes during treatment by measuring a line from midpoint of SN to point $\mathrm{B} .{ }^{(5)} \mathrm{He}$ demonstrated actual measurements of two cases from his study ( fig.2) . He explained that an angle is a figure formed by two lines diverging from the same point. The longer these lines are extended the further apart they become in relation to each other. So as the distance between the nasion to points $\mathrm{A}$ and $\mathrm{B}$ increases in length, the linear measurements between the latter also increases. However, the ANB angle itself is unaffected by the length of the sides forming it. In this way it is possible for two patients to have identical ANB values but the with different horizontal distance values between $\mathrm{A}$ and $\mathrm{B}$ according to the length of their face. 
A
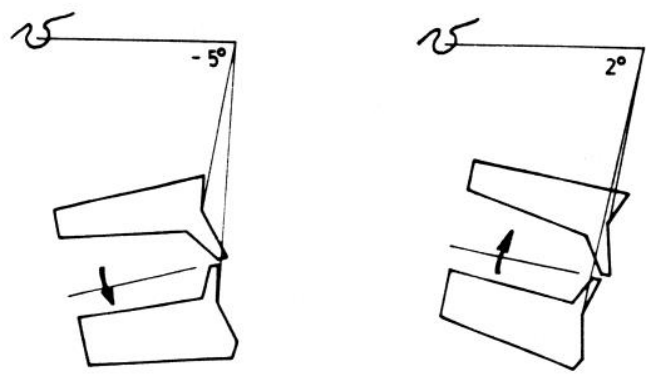

B

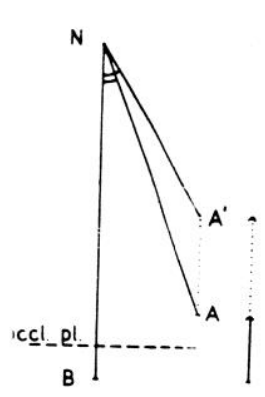

$N^{\prime}$

C<smiles>[H]</smiles>
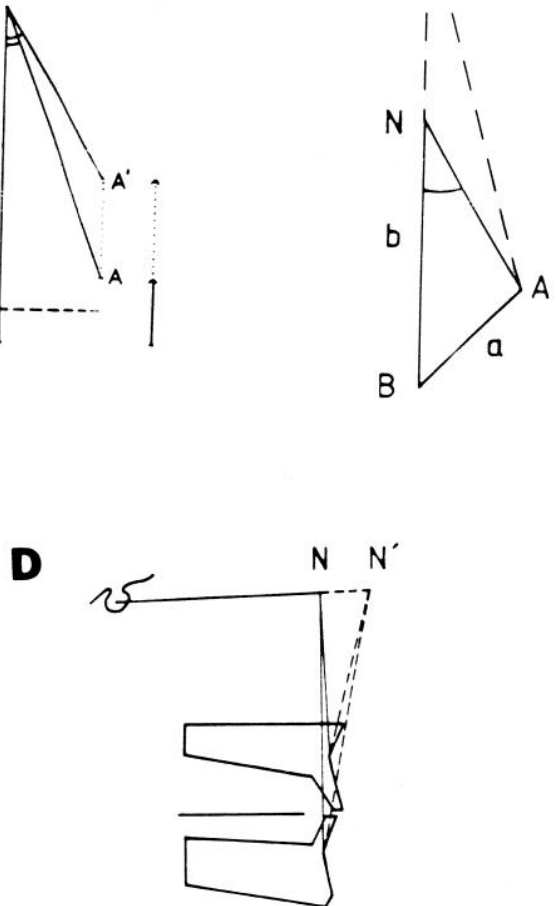

Fig.1. Qualitative illustration of the effects on angle ANB of changing the size of one parameter and holding the others constant. A, Opening rotation of the occlusal plane. $\mathrm{B}$, Increasing the dental height. C, Increasing the length N to B. D, Anteroposterior position of $\mathrm{N}$ with respect to points $\mathrm{A}$ and $\mathrm{B}$. 

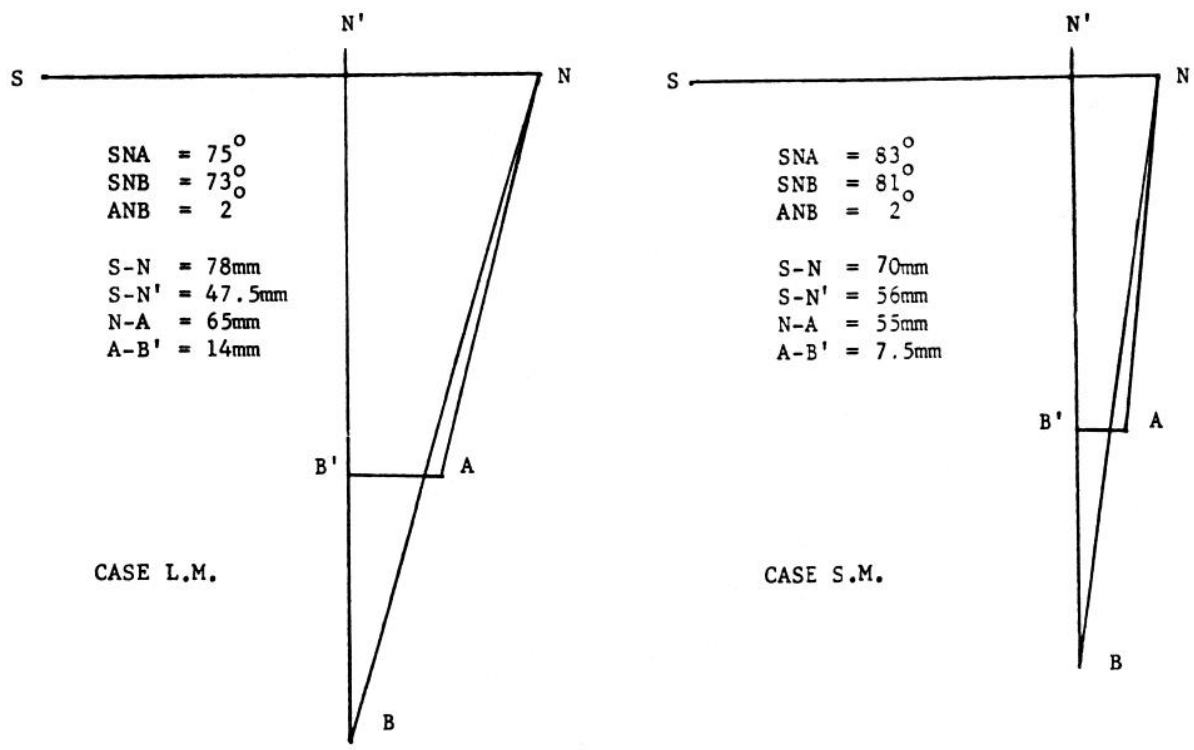

Fig. 2: Actual measurements taken from two cases, Note that although both patients have identical ANB differences, the relationship of point A to point $\mathrm{B}$, expressed by the measurement A-B, is quite different. Also apparent is the influence exerted by the relative positions of nasion, point $\mathrm{A}$, and point $\mathrm{B}$ and the manner in which they establish facial divergence. Patient L. M. could be expected to require more vigorous treatment to effect an apical base reduction than patient S. M., even though the ANB indicated that both cases were equal in this respect.

A study done by Jarvienen ${ }^{(8)}$ to evaluate the appropriateness of the ANB angle as measure of the sagittal apical base relationship, revealed that the difference in the means of the ANB angle was statistically significant between the separate malocclusion samples. However, the range of individual variation was large and angles of equal size could be found in all malocclusion classes, thus the interpretation of the ANB angle did not always confirm to the clinical Angle classification.

It was further suggested that points $\mathrm{S}$ and $\mathrm{N}$ as cephalometric landmarks require caution, because many variations are associated with these points relating to age, sex and individual differences. ${ }^{(9)}$ Consequently a measurement independent of cranial reference planes would be a desirable adjunct in determining the apical base relationships. ${ }^{(2)}$ 
A second widely used measurement, the Wits appraisal, was introduced by Jacobson to overcome problems related to the ANB angle. ${ }^{(3,10)}$ However as the Wits appraisal relates points $\mathrm{A}$ and $\mathrm{B}$ to the functional occlusal plane; two problems occur. First, accurate identification of the occlusal plane is not always easy or accurate ${ }^{(11)}$ especially in the mixed dentitions and open bite patients. Second, any change in the angulation of the functional occlusal plane, caused by either normal development of the dentition or orthodontic intervention can influence the Wits appraisal ${ }^{(12,13)}$. Therefore, Wits appraisal might be of limited value because it reflects changes in the occlusal plane instead of pure anteroposterior changes of the jaws.

Nanda and Merrill ${ }^{(14)}$ suggested a measurement based on the Palatal plane. However, the variable inclination of this plane makes it difficult to establish mean values for the norm.

A specific measurement for apical base difference should be independent of cranial reference planes or dental occlusion. The Beta Angle ${ }^{(15)}$ was developed as it does not depend on the previous factors and it would be valuable whenever previously established cephalometric measurements such as ANB angle and Wits appraisal, cannot be accurately used because of their dependence on various factors.

The Beta angle ${ }^{(15)}$ uses three skeletal landmarks, point $\mathrm{A}$, point $\mathrm{B}$, and the apparent axis of the condyle point $\mathrm{C}$ to measure an angle that indicates the apical base relationship in the sagittal dimension (fig.3).

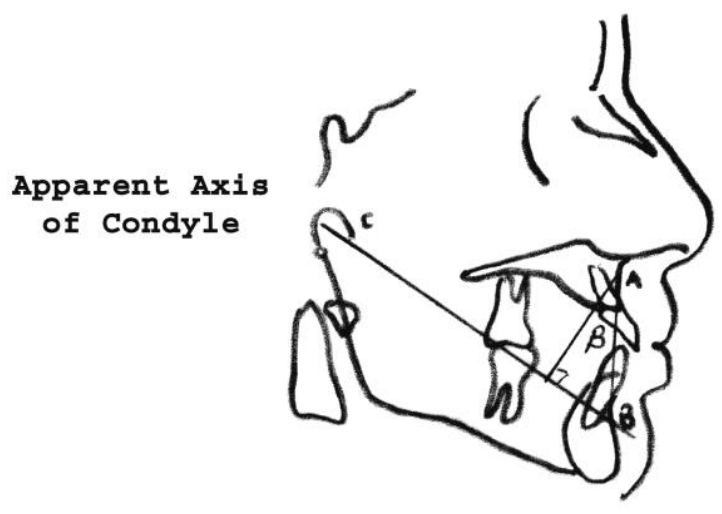

Fig.3: Beta angle 
Point A: Deepest midline point on the maxilla between the anterior nasal spine and the prosthion.

Point B: Deepest midline point on the mandibular symphysis between the infradentale and pogonion.

Point C: Center of the head of the condyle.

\section{The angle is formed by the following 3 lines:}

C-B line connecting B point to the center of the condyle, A-B line and a line perpendicular from A point to C-B line. The Beta angle is the angle between the perpendicular line and the A-B line.

The aim of this study was to find the mean and the standard deviation values for this angle in an Egyptian population sample having different sagittal skeletal patterns and to compare the results of this study with the study done at Tufts University.

\section{Material and Methods:}

Files belonging to 120 patients with various skeletal patterns were selected from the orthodontic clinic of Alexandria University and one private orthodontic practice in Alexandria.

Pretreatment cephalometric x-rays were retraced to measure angle ANB, Wits appraisal, Beta angle and the XY axis angle. Measurements were measured twice and the mean values calculated. There were 40 class I cases, 45 class II cases and 35 class III cases.

The $\mathrm{XY}$ axis was included to define the skeletal pattern in the vertical dimension as the vertical pattern affects the ANB angle dramatically.

For Class I subjects chosen patients had ANB angle greater or equal to 2 degrees and smaller or equal to 4 degrees, Wits appraisal between $+1 \mathrm{~mm}$ and $-3 \mathrm{~mm}$ XY axis angle 93 degrees + or -5 degrees. 27 cases out of the 40 selected met the previous criteria others were excluded mainly due to deviations in the vertical dimension.

For Class II subjects chosen patients had ANB angle greater than 4 degrees. Wits appraisal more than $1 \mathrm{~mm}$ and a class II profile. 34 cases out of the 40 selected met the criteria and the rest were excluded.

For the Class III subjects chosen patients had ANB angle less than or equal to 1 degree. Wits appraisal less than or equal to -4 and a class III profile. 27 cases 
were selected out of the 35 cases chosen and the rest of the cases were excluded as they didn't fulfill the previous criteria.

The final 88 cases selected were between 12 and 18 years of age .The Beta angle was measured for each patient in the three groups twice and the mean values calculated.

\section{Statistical analysis:}

Comparison of the mean values of Beta angles among the classes was performed using ANOVA. Posthoc analysis was performed using Scheffe's test.

Receiver Operating Characteristic curves (ROC analysis) was done to determine the Beta angle cut off points for classes II and III. Sensitivity and specificity were determined for the success of the Beta angle in identifying classes II and III.

Bars and ROC curves were used for graphical presentation of the data. Statistical analysis was performed using SPSS version 13 and Med Calc software.

\section{RESULTS}

Table I shows that the mean value for the Beta angle in skeletal class I in the chosen Egyptian sample was 32.2 degrees with a standard deviation of 1.5 degrees. The mean value for skeletal class II was 25.1 degrees with a standard deviation of 3 and the mean value for skeletal class III was 41.8 degrees with a standard deviation of 5 . (table I).

The ANOVA showed that the three groups are statistically significant

Receiver Operating Characteristic (ROC) curves (figure 4,Table II) showed that a Beta angle less than or equal to 29 degrees has a $97.1 \%$ sensitivity and $100 \%$ specificity for discriminating class II from class I skeletal patterns. A Beta angle greater than 34 degrees has a $100 \%$ sensitivity and specificity for discriminating class III from class I skeletal patterns.

Thus the cutoff points between class I and II is approximately 29 degrees and between class I and III approximately 34 degrees. So a patient with Beta angle less or equal to 29 degrees is considered to be a class II skeletal pattern and between 29 and 34 degrees a class I skeletal pattern. While patients with a Beta angle above 34 degrees are considered to have class III skeletal pattern. 
Egyptian

Orthodontic Journal

Table 1: Comparison of Beta angle measurement among the three classes:

\begin{tabular}{|l|c|c|c|}
\hline & $\begin{array}{c}\text { Class I } \\
\mathbf{n = 2 7}\end{array}$ & $\begin{array}{c}\text { Class II } \\
\mathbf{n = 3 4}\end{array}$ & $\begin{array}{c}\text { Class III } \\
\mathbf{n = 2 7}\end{array}$ \\
\hline Mean & 32.22 & 25.18 & 41.80 \\
\hline SD & 1.50 & 3.09 & 5.01 \\
\hline Mean \pm SD & $29.22-35.33$ & $19-31.36$ & $31.78-51.82$ \\
\hline F test & & 172.31 \\
P value & & $0.0001^{*}$ \\
\hline
\end{tabular}

$*$ : Statistically significant $\mathrm{P} \leq 0.05$

Pairwise comparison using Scheffe's test shows statistically significant differences between all pairs.

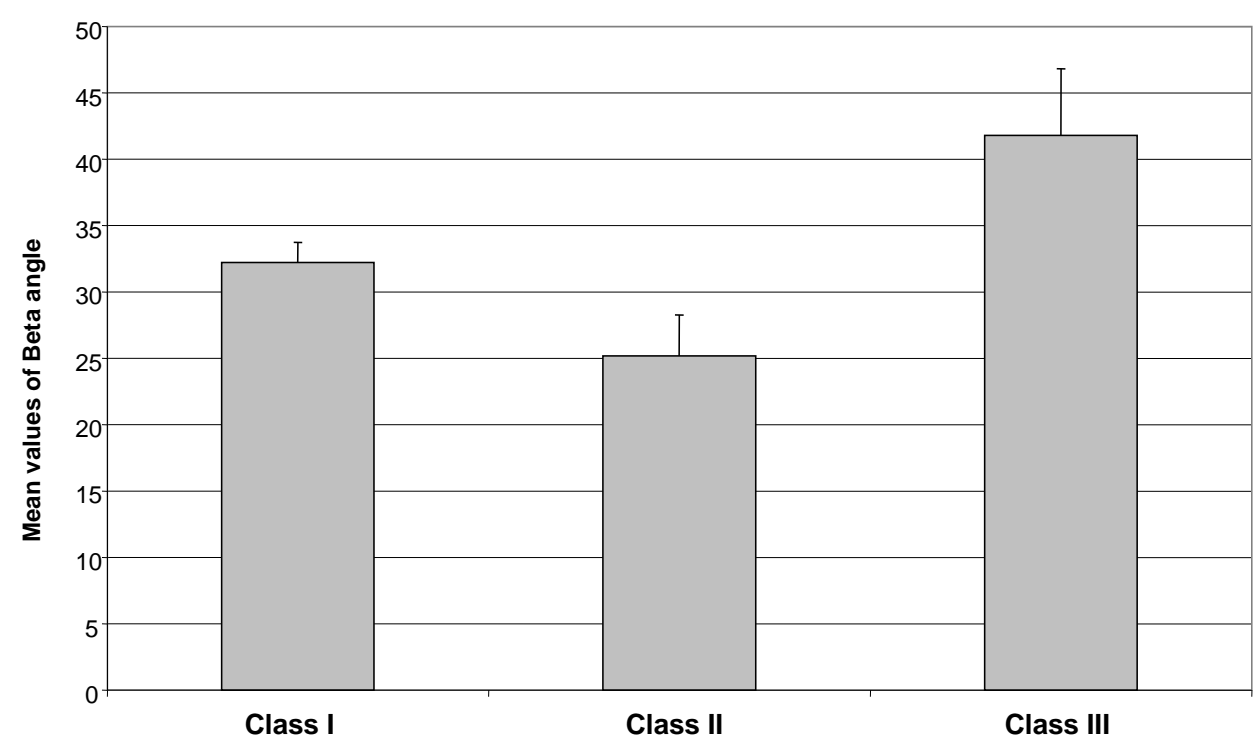

Mean values of Beta angle in the three classes 
Egyptian

Orthodontic Journal

Table 2: Cut off point, sensitivity and specifity of Beta angle in identification of different classes

\begin{tabular}{|l|c|c|c|}
\hline & Cut point & Sensitivity & Specificity \\
\hline Class II & $\leq 29$ & 97.1 & 100 \\
\hline Class III & $>34$ & 100 & 100 \\
\hline
\end{tabular}

NS: Not statistically significant

*: Statistically significant $\mathrm{P} \leq 0.05$
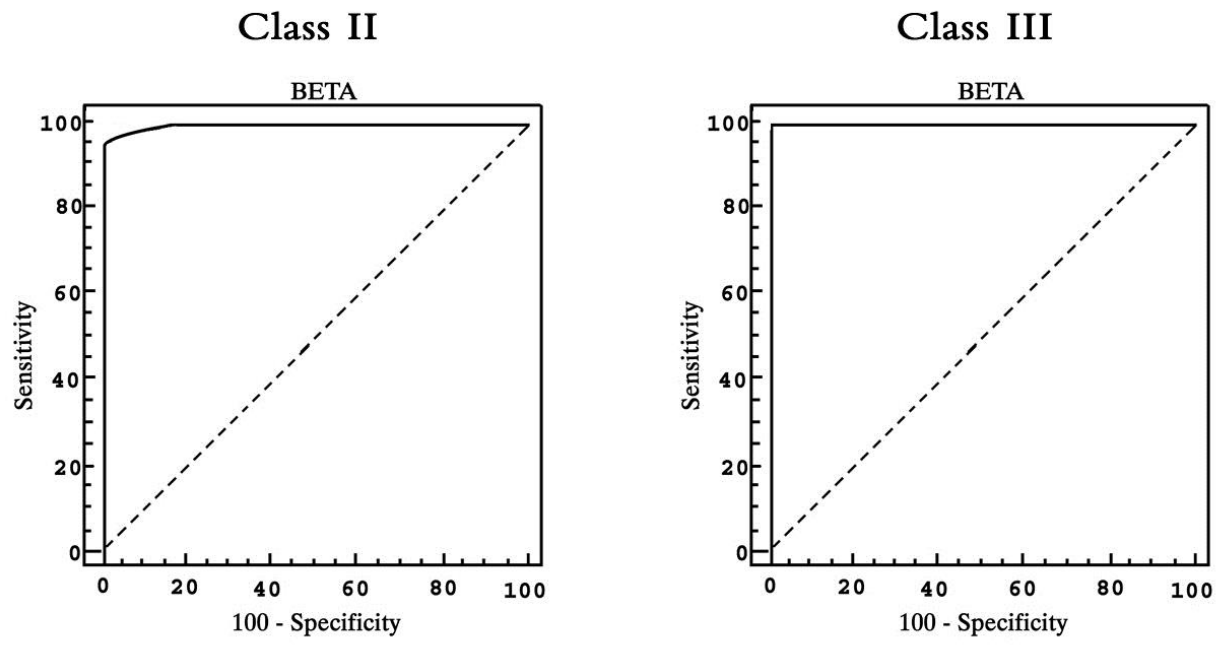

Fig. 4: ROC curves for Beta angle discriminating classes II and III

\section{DISCUSSION}

Cephalometric analysis remains a very important tool in the orthodontic diagnosis and treatment planning, however sagittal jaw relations are difficult to evaluate. Distinctly different sagittal jaw relations may have the same measurement values according to the methods of analysis currently in use $\mathrm{e}^{(14)}$.

The ANB angle commonly used to study and define the relationship between the maxillary and the mandibular skeletal bases is affected by a variety of factors which complicates the correct interpretation of such an angle. 
The Beta angle does not depend on cranial landmarks or functional occlusal plane. It uses three points located on the jaws: point A, point $\mathrm{B}$ and point $\mathrm{C}$ (center of the condyle). This angle reflects changes in the jaws alone and remains stable even when the jaws are rotated ${ }^{(15)}$.

Figure 5 shows point $\mathrm{B}$ rotating backwards and downwards rotating the $\mathrm{C}-\mathrm{B}$ line in the same direction. The A-B line is rotating in the same direction so the Beta angle is relatively stable. Thus this angle reflects true changes in the anteroposterior jaw relation and can be relied upon in diagnosis and treatment planning.

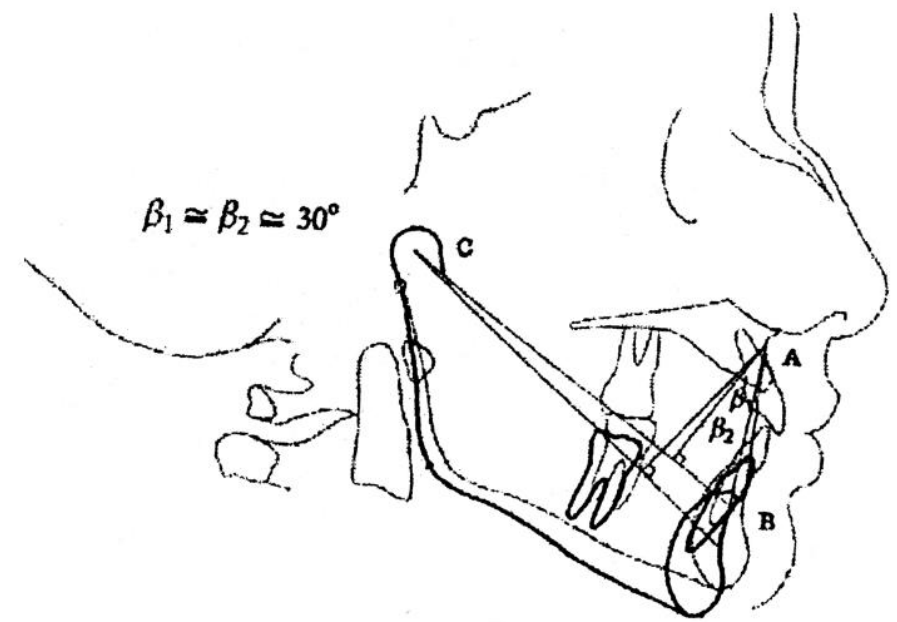

Fig. 5: Beta angle remains relatively stable even when jaws are rotated.

A difficulty using this angle might be in precisely locating the center of the condyle. However according to Mc Namara ${ }^{(16)}$ the advantage of locating the center of the head of the condyle versus the condylon point, is that very precise tracing of the contour of the condyle is not really necessary. The clinician can locate the approximate center with a minimum error in the Beta angle as long as the point is within $2 \mathrm{~mm}$ of its actual location.Figure $6^{(15)}$ shows that if the true center is at $\mathrm{C}$, but the clinician locates it within a circle of $2 \mathrm{~mm}$ radius, then the Beta angle is affected less than 1 degree which makes it still acceptable. 


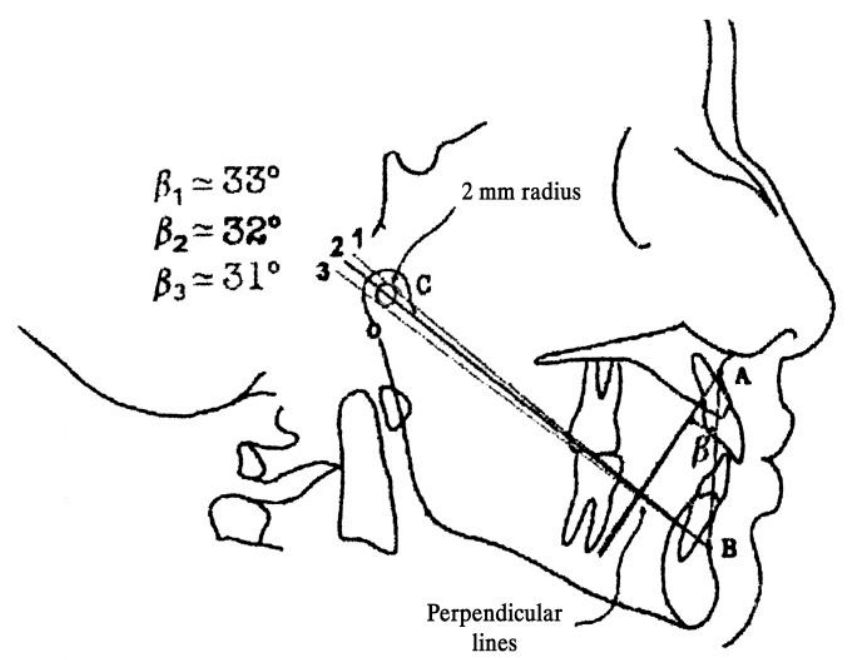

Fig. 6: If clinician can locate point that falls in circle between lines 1 and 3, Beta angle is affected less than $1^{\circ}$.

Another fact is that the Beta angle only determines the skeletal pattern but does not determine which jaw is prognathic or retrognathic, which should be diagnosed from other cephalometric data.

This study was done to define Egyptian norms for the Beta angle to be able to use it with the Alexandria cephalometric analysis on Egyptian population. Comparing the results of this study to the Beta angle measures at Tufts University, the mean value of the Beta angle was 32.2 degrees for class I in the Egyptian population sample instead of 31.1 degrees. For class II the mean value for the Beta angle was 24.1 degrees instead of 24.5 degrees and for class III the mean value was 41.8 degrees for the Egyptian population sample instead of 40 degrees.

The cut off point between classes I and II is 29 degrees for the Egyptian population instead of 27 degrees and the cut off point between classes I and III is 34 degrees for the Egyptian sample instead of 35 degrees. This minute difference in the values of the beta angle is due to variations in the skeletal pattern in different populations. 
It is concluded that Egyptian subjects with Beta angle greater than 29 degrees and smaller than or equal to 34 degrees have a class I pattern, those with Beta angle smaller than or equal to 29 degrees to have a skeletal class II pattern and those with Beta angle greater than 34 degrees have a class III skeletal pattern.

The Beta angle can be thus added as an extra diagnostic measurement in cephalometrics to help in diagnosing the skeletal pattern of the patient away from the factors that would tend to camouflage this pattern.

\section{REFERENCES}

1- Beatly EJ: A modified technique for evaluating apical base relationship. Am J Orthod. 1975, 68:303-315.

2- Bishara SE, Fahl JA, Peterson LC: Longitudinal changes in the ANB angle and the Wits appraisal; clinical implications. Am J Orthod. 1983, 84:133-139.

3- Jacobson A: The Wits appraisal of jaw disharmony. Am J Orthod. 1975, $67: 125-138$.

4- Jarvinen S: A comparison of two angular and two linear measurements used to establish sagittal apical base relation. Eur. J Orthod. 1981, 3:131-134.

5- Taylor CM: Changes in the relationship of Nasion, point $A$, and point $B$ and the effect upon ANB. Am J Orthod. 1969, 56:143-163.

6- Binder RC: The geometry of cephalometrics. J. of Clinical Orthod. 1979, 13:258-263.

7- Wolfram Hussels and Ram S. Nanda: Analysis of factors affecting ANB. Am J Orthod. and Dentofacial Orthopaedics. 1984, 85:411-423.

8- Jarvinen S: An analysis of the variation of the ANB angle: A statistical appraisal. Am J of Orthod. and Dentofacial Orthop. 1985, 87:144-146.

9- Enlow DH: Handbook of facial growth, ed. 2, Philadelphia 1982. W.B. Saunders company.

10- Jacobson A: The application of the Wits appraisal. Am J Orthod. 1976, 70:179-89.

11- Haynes S, Chau M: The reproducibility and repeatability of the Wits analysis. Am J Orthod. and Dentofacial Orthop. 1995,107:640-7. 
12- Rushton R, Cohen AM, Linney FD: The relationship and reproducibility of angle ANB and the Wits appraisal. Br. J Orthod. 1991,18:225-31.

13- Sherman SL, Woods M, Nanda RS: Longitudinal effects of growth on the Wits appraisal. Am J Orthod. Dentofacial Orthop. 1988, 93:429-36.

14- Nanda RS and Merrill RM: Cephalometric assessment of sagittal relationship between maxilla and mandible. Am J Orthod. and Dentofacial Orthop. 1994, 105:328-44.

15- Baik CY, Ververidou M: A new approach of assessing sagittal discrepancies: The Beta angle. Am J Orthod. Dentofacial Orthop. 2004,126:100-5.

16- Mc-Namara JA Jr. A method of cephalometric evaluation. Am J Orthod. 1984, 86:449-69. 